\title{
Comparison of Pairwise Dissimilarity and Projective Mapping Tasks With Auditory Stimuli
}

\author{
M. J. VOWELS* AND R. MASON, AES Member \\ (m.j.vowels@surrey.ac.uk) \\ (r.mason@surrey.ac.uk) \\ University of Surrey, Guildford, United Kingdom
}

\begin{abstract}
Two methods for undertaking subjective evaluation were compared: a pairwise dissimilarity task (PDT) and a projective mapping task (PMT). For a set of unambiguous, synthetic, auditory stimuli, the aim was to determine the following: whether the PMT limits the recovered dimensionality to two dimensions; how subjects respond using PMT's two-dimensional response format; the relative time required for PDT and PMT; and hence, whether PMT is an appropriate alternative to PDT for experiments involving auditory stimuli. The results of both Multi-Dimensional Scaling (MDS) analyses and Multiple Factor Analyses (MFA) indicate that, with multiple participants, PMT allows for the recovery of three meaningful dimensions. The results from the MDS and MFA analyses of the PDT data, on the other hand, were ambiguous and did not enable recovery of more than two meaningful dimensions. This result was unexpected given that PDT is generally considered not to limit the dimensionality that can be recovered. Participants took less time to complete the experiment using PMT compared to PDT (a median ratio of approximately 1:4), and employed a range of strategies to express three perceptual dimensions using PMT's two-dimensional response format. PMT may provide a viable and efficient means to elicit up to 3-dimensional responses from listeners.
\end{abstract}

\section{INTRODUCTION}

Subjective testing is important for a number of contexts such as product development in the consumer market, auditory assessment of sound quality, and psychological research. Many methods have been developed, each with their own relative advantages and disadvantages [1]. Two such methods are considered in the current study: The Pairwise Dissimiliarity Task (PDT), which is a traditional method requiring participants to rate the perceived differences between pairs of stimuli [1]; and the Projective Mapping Task (PMT), which requires participants to position icons or objects representing each stimulus in a two-dimensional space according to the relative perceived similarities and differences between the stimuli [2]. Projective mapping in particular is becoming increasingly popular in the sensory sciences [3].

\subsection{The PDT}

In the PDT, participants compare each stimulus with each of the other stimuli, one pair at a time [1]. While this method directly generates a set of dissimilarity data between each and every pair of stimuli, the experiment duration can be

*To whom correspondence should be addressed. prohibitively long. The number of comparisons $(C)$ to be undertaken by the subject can be calculated using $C=n(n$ $-1) / 2$, where $n=$ the number of stimuli in the stimulus set [4]. Hence, the number of comparisons is proportional to $n^{2} / 2$, meaning that the number of comparisons increases significantly as the number of stimuli increases. For example, for 5 stimuli, only 10 comparisons are required, but for 15 stimuli, 105 comparisons are required. This is compounded by the fact that, for common types of analysis such as Multi-Dimensional Scaling (MDS), a minimum number of stimuli per dimension is required to achieve stable results. Schiffman and Knecht advised that 18 stimuli be used for three dimensions (i.e., six per dimension) [5], whereas Davison et al. suggested that five stimuli per dimension is acceptable depending on the nature of the stimuli [6]. In addition to the concomitant expense associated with long experiments, a further consequence is participant fatigue, which may affect the quality of the data [7]. To combat fatigue, tests may be split into a number of sessions. Even so, the participants may experience a learning curve as their rating strategy evolves throughout the course of the test [8], and this lack of strategic consistency may also adversely affect the data. PDT does not usually allow participants to revisit, review, or revise their decisions, so ratings cannot be updated to reflect this later learning. 
Therefore, there is a need for quicker, more efficient methods for collecting dissimilarity data, methods that potentially also allow participants to revisit, review, and revise their responses during the test [9].

\subsection{The PMT}

PMT, of which "Napping" is a specific example [10], is a relatively new psychological measurement method introduced in the mid-1990s [11]. In PMT, the participant is required to position markers that represent the stimuli in a two-dimensional space according to how they perceive the stimuli to be related to each other [2]. Two stimuli are placed close to each other if they are perceived as similar and far from one another if they are perceived as different. A participant's PMT arrangement is recorded using a coordinate system, from which the Euclidean distances between each of the stimuli can be measured [12]. Scavone et al. explain how PMT is an effective alternative to PDT [13], especially with regards to reducing subject fatigue. In addition, it is argued that the ability to adjust the responses and revisit previously decided inter-stimulus relationships increases the consistency of judgments.

Risvik et al. consider that PMT is sufficiently intuitive to be used in applications with children [14], with whom less-developed language abilities may be an obstacle in traditional scaling exercises: data collection methods should be easy to understand and not laborious for the participants. Research by King et al. indicated that some participants were hesitant when first presented with PMT [12], though this hesitance was reported to have soon evaporated, and most of the untrained assessors apparently found the task to be "interesting and less difficult than they had anticipated."

The main disadvantage of PMT is that the resulting data is inherently restricted to two dimensions [4]. Analysis of a single PMT dissimilarity matrix can therefore only reveal the original two-dimensional graphical arrangement made by the subject. In order for MDS or MFA to recover more than two dimensions, an analysis of a number of PMT responses is required [4].

\subsection{Discussion}

Despite PDT being the traditional method of deriving data for MDS, there are a number of disadvantages to this technique as discussed above. On the other hand, there is no indication that data acquired using PDT is limited in recoverable dimensionality [15]. In contrast to PDT, there has been some debate as to whether data acquired using PMT can be analyzed to recover more than two dimensions [11]. Using visual stimuli, Nestrud and Lawless found that by combining results over a number of participants, three dimensions could be interpreted [11]. Inspection of the individual results indicated that this was partially due to participants attending to differing pairs of dimensions from the three in which the stimuli varied (e.g., rating the differences in color and shape while ignoring size). Some subjects attempted to find alternative solutions to represent the three dimensions, including representing a dimension using the radius from the center of the response sheet.

The majority of the studies outlined above have employed PMT for either visual stimuli or in the area of food science; little study has been undertaken into PMT for psychoacoustics. One example of the technique in audio [16] utilized PMT to evaluate loudspeaker perceptual quality; when this paper was presented, an audience member questioned whether PMT can be used to recover more than two meaningful dimensions for auditory stimuli. In addition, it is of interest to discover the response methods attempted by subjects and whether they find PMT overly restrictive. Finally, it would be useful to determine the relative time required for PDT and PMT methods for auditory experiments, to determine whether PMT is significantly more efficient than PDT.

Therefore, this paper compares PDT and PMT to determine the following: whether more than two dimensions can be recovered from data derived using PMT; how participants respond to PMT's inherently two-dimensional response format; the relative time required to complete the two tasks; and, hence, whether PMT is a viable method for collecting data for experiments involving auditory stimuli.

\section{METHOD}

\subsection{Stimuli}

In order to measure the relative effectiveness of PDT and PMT for Multi-Dimensional Scaling (MDS) and Multiple Factor Analysis (MFA), a set of stimuli were synthesized. Three main aspects were considered: the number and nature of the dimensions and the number of stimuli.

\subsubsection{Number and Nature of Dimensions}

To establish whether the tasks limit the number of dimensions that may be recovered to fewer than three, the stimuli needed to vary in at least three unambiguous perceptual dimensions. It was important that these dimensions were perceptually orthogonal (and clearly distinct) so that any failure of the analysis to recover all three dimensions would be due to limitations associated with the task and/or analysis, rather than because any of the participants failed to identify or perceive a particular dimension. For this reason, the participants were intentionally primed with knowledge of the number and nature of the perceptual dimensions of the stimuli. This is dissimilar to the usual application of these methods, in which determining the number and arrangement of the perceptual dimensions are the aims of the experiment; in this case, the aim was to discover whether the experimental method limited the determination of the known perceptual dimensions.

For perceptual simplicity and clarity, the stimuli were based on sinusoidal tones, and the perceptual dimensions were determined from a combination of prior research and pilot experimentation. Based on the research of Letowski, two perceptual dimensions selected represented the two high-level groupings of timbral and spatial characteristics [17]. An independent variable (IV) for each was varied: 
for timbre, amplitude modulation/"tremolo" was varied; for spatial characteristics, the interaural presentation of each stimulus was varied. The third perceptual dimension selected was pitch; for this, the frequency of the tone was varied. It is known that there is often some interaction between perceptual dimensions (e.g., [18] and [19]); however, a pilot experiment confirmed that participants could clearly and separately identify the three perceptual dimensions used in this experiment without difficulty.

\subsubsection{Number of Stimuli}

As discussed above, there is some discussion about the number of stimuli that are required in order to enable stable results upon analysis. In order to maximize the chance of successful recovery of three dimensions, 18 stimuli in total were created (six stimuli per dimension). A full factorial design for the three IVs was employed, with two IVs having three levels, and the third IV having only two levels. As such, a full factorial design required $3 \times 3 \times 2=18$ stimuli to include all possible combinations of the independent variables. The IVs were as follows: pitch (frequencies of 180,240 , or $300 \mathrm{~Hz}$ ); interaural presentation (left ear only, diotic, or right ear only); and tremolo (tremolo on or off).

\subsection{Stimulus Presentation}

The experiment was conducted using the SonicMapper software [13]. For PDT, this presented the participant with buttons to play each stimulus to be compared, plus a slider to indicate the similarity (from "low similarity" to "high similarity"). For PMT, this presented each stimulus as a box on a computer screen, and the subject could use the mouse to play each stimulus and position them on the screen as required.

This software ran on an Apple Macbook Pro with an Apogee Duet audio interface, which was connected to a pair of AKG Q701 headphones. The experiment was undertaken in an environment free from significant visible and audible distractions.

\subsection{Procedure}

Each participant was given a set of instructions explaining the nature of the task that he or she was to undertake. In order to maximize the chance of being able to recover three dimensions, the instructions informed the subjects that the stimuli varied in stimulus frequency, interaural presentation, and tremolo on/off state so that they were fully aware of the nature and dimensionality of the stimuli.

Each test was preceded by a familiarization exercise: all 18 stimuli were presented in a random order, and the participants were encouraged to listen to all and consider the ways in which they were similar or different. This was followed by a short practice test to help familiarize the participants with their respective task. This process took less than 5 minutes for each listener. Subjects were encouraged to take their time both for the familiarization exercise as well as the real test, and a stopwatch was used to record the time taken for each to complete the main task.

\subsection{Participants}

The listening panel comprised 24 participants, all of whom were undergraduate audio engineering and music (Tonmeister) students at the Institute of Sound Recording at the University of Surrey, United Kingdom. They may be considered "discerning" participants with prior listening test experience, training in critical listening, and strong musical ability. Neher et al. indicate that while more experienced participants may be more consistent, there is no guarantee that they will be representative of the "average" participant [20]. However, it was felt that using "discerning" participants with prior listening test experience would maximize the chance of recovering three dimensions. The 24 participants were randomly assigned to one of the two tasks: 12 participants undertook PDT, and the other 12 undertook PMT. Although no evaluation was undertaken to check the homogeneity of the two groups, we ensured that all listeners could identify the perceptual dimensions of the stimuli.

\section{RESULTS}

The data included the $x, y$ co-ordinates from the PMT, the dissimilarity ratings (direct dissimilarity ratings for PDT and measured Euclidean distances for PMT), and the duration that it took each subject to complete the relevant task. Two types of analysis were undertaken: MDS and MFA. The details of, and results from, these analyses will now be discussed in turn.

\subsection{Analysis-MDS}

In order to analyze the data from such methods, MDS is often employed in order to recover the underlying dimensionality of the stimuli [21-23]. MDS is a statistical data analysis technique that aims to determine the multidimensional spatial/graphical representation of the distances, differences or dissimilarities between underlying dimensional constructs in data [24].

The dissimilarity ratings for PDT and the measured Euclidean distances for PMT were analyzed using MDS algorithm ALSCAL using SPSS24 by IBM. For individual result sets (each of the participants' responses analyzed separately), classical MDS (CMDS) was employed, and for the combined analysis of data from all participants, weighted MDS (WMDS) was employed. The MDS algorithm ALSCAL was used, as it can undertake both CMDS and WMDS. This way, results from individual participants, as well the results from the two groups, may be analyzed and compared. The data resulting from PDT cannot be assumed to be Euclidean [25], so both metric and nonmetric CMDS (for individuals) and WMDS (for all participants together) were undertaken.

The main factor of interest in the experiment was the number of meaningful dimensions that could be recovered from the data. The number of resulting dimensions is typically determined based on measures of "goodness of fit" that indicate how successfully an MDS model fits the original data against different dimensionalities [8]. In the 

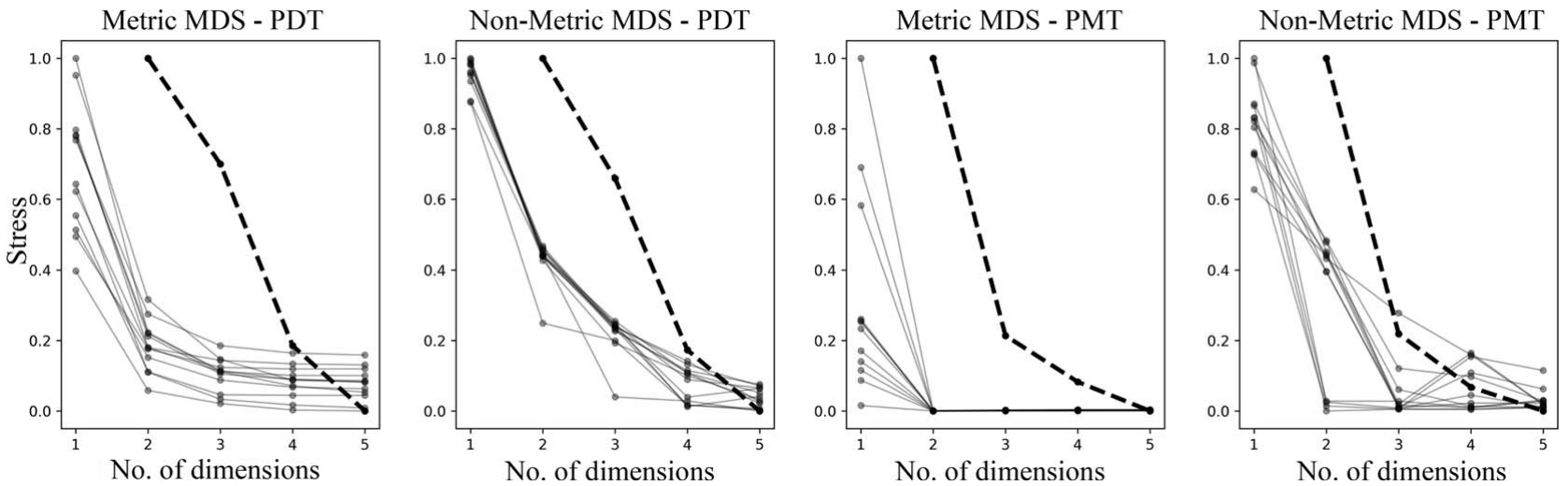

Fig. 1. Normalized Kruskal's Stress for the metric and nonmetric Multi-Dimensional Scaling (MDS) analyses of the pairwise dissimilarity task (PDT) and projective mapping task (PMT) data, for each participant's responses (faded lines) and all participants together (dashed lines).

case of the ALSCAL algorithm, the predominant metric is "Kruskal's Stress" [20]. A distinct change (a knee) in the gradients of the curves for Stress, as the number of dimensions is increased, can be used to identify the dimensionality of the data [15]. It is worth noting that such metrics as Stress can sometimes be misleading, and the perceptual relevance of the resulting dimensions may also be interpreted by visual examination of the solution [20]. Specifically, the perceptual structure of the stimuli in this experiment is expected to conform to three dimensions: stimulus frequency, interaural presentation, and presence of tremolo. Hence, in addition to the measures of goodness of fit, the resulting MDS structures were also visually examined, ensuring that the MDS solutions were rotated through all permutations (as the resulting axis orientation is arbitrary), to determine whether the represented dimensions were meaningful and met the expectations for the underlying perceptual dimensions.

\subsection{PDT Results}

The PDT data were analyzed separately for each participant using both metric and nonmetric CMDS. An overview of the Stress values for solutions with 1 to 5 dimensions for each participant's results is shown in Fig. 1 (the faded lines in the two leftmost plots). For the metric analysis, it can be seen that there is some indication of a knee at two dimensions (although we know the participants could perceive three dimensions), and for the nonmetric analysis, there is no clear knee indicated for most participants. Hence, it is difficult to confidently ascertain how many meaningful dimensions have been recovered using the metric and nonmetric CMDS analyses.

The resulting nonmetric CMDS 3-dimensional solutions were also visually inspected. The results for the participant that showed the most likely knee point for a 3-dimensional solution is shown in Fig. 2. It can be seen that there are clear groups in dimensions 1 and 2 separated by stimulus
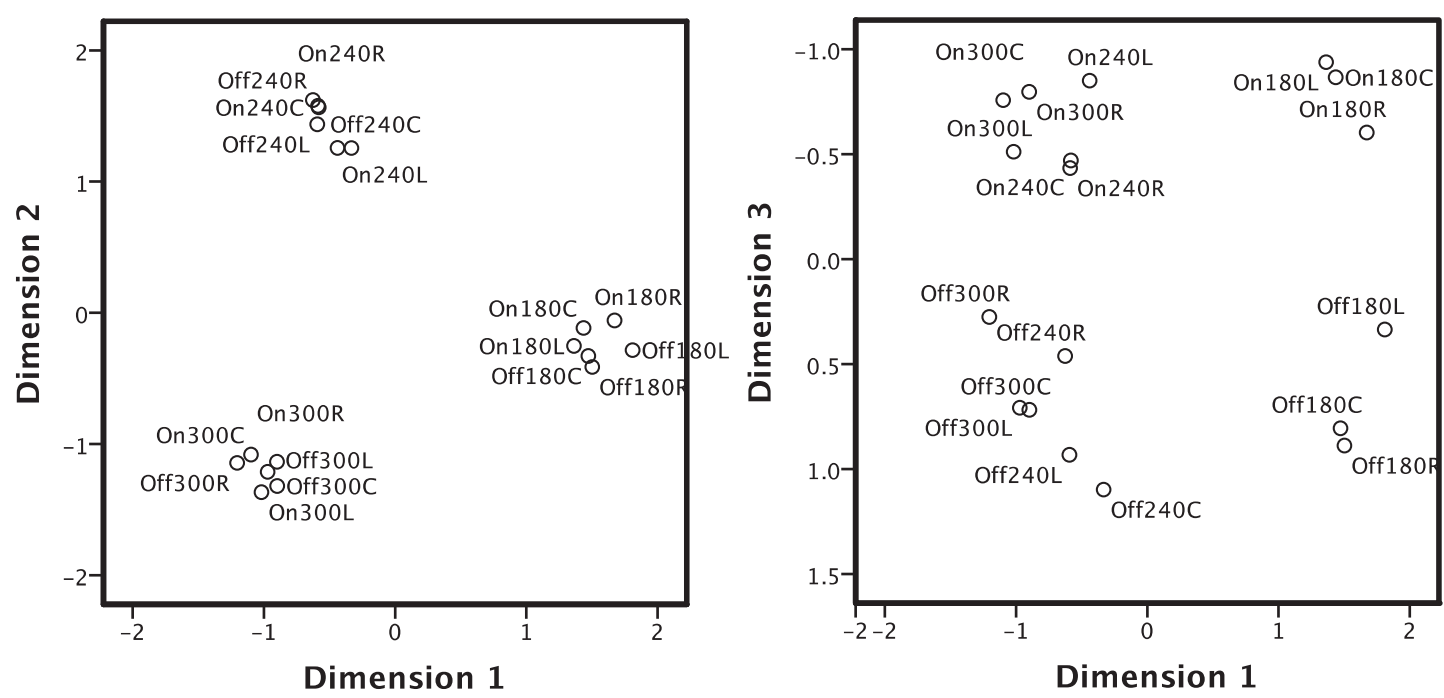

Fig. 2. The nonmetric classical MDS (CMDS) 3-dimensional solution for participant 9's pairwise dissimilarity task (PDT) data, showing dimension 1 vs. dimension 2 in the left plot and dimension 1 vs. dimension 3 in the right plot. The points are labelled with a stimulus code: On/Off refers to the tremolo; 180/240/300 refers to the frequency; and L/C/R refers to the interaural presentation. 

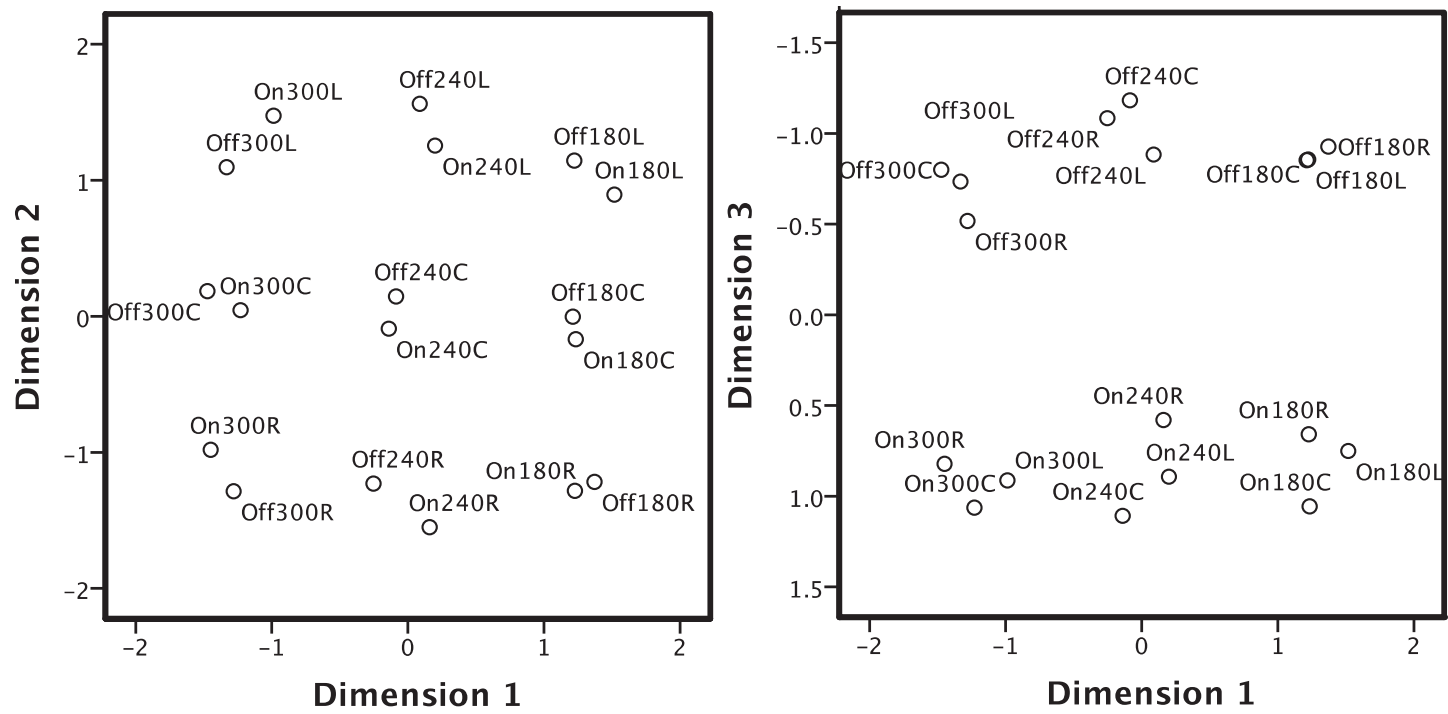

Fig. 3. The nonmetric classical MDS (CMDS) 3-dimensional solution for participant 12's pairwise dissimilarity task (PDT) data, showing dimension 1 vs. dimension 2 in the left plot and dimension 1 vs. dimension 3 in the right plot. The points are labelled with a stimulus code: On/Off refers to the tremolo; 180/240/300 refers to the frequency; and L/C/R refers to the interaural presentation.

frequency and in dimension 3 by tremolo on/off. However, the interaural presentation of each stimulus is not clearly differentiated on these three dimensions (or when the dimensions are rotated). It was found that the data from only one participant could be meaningfully represented in three dimensions in the CMDS analysis, and this is displayed in Fig. 3. It can be seen that the stimulus frequency is spread across dimension 1, the interaural presentation is spread across dimension 2, and the tremolo on/off is spread across dimension 3. Further investigation showed that for most participants, a dimension relating to the interaural presentation could not be derived from the data.

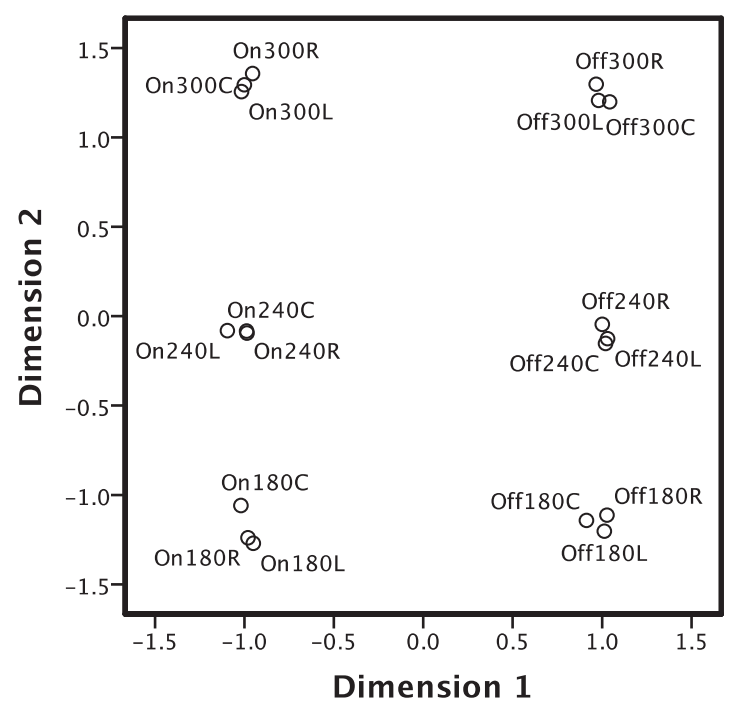

The PDT data from all participants were then analyzed using metric and nonmetric WMDS, and the Stress values for solutions with 2 to 5 dimensions are shown by the dashed line in the two leftmost plots in Fig. 1. As with the individual participant results, there is no clear knee point.

The WMDS solutions were also visually inspected, and the nonmetric three-dimensional solution is shown in Fig. 4. It can be seen that the groupings/clusters of stimuli associated with dimension 1 correspond with tremolo on or off from right to left, respectively. The vertical groupings/clusters associated with dimension 2 correspond to the stimulus frequency. However, the clusters in dimension 3 also correspond with the stimulus frequencies $(240 \mathrm{~Hz}$ low,

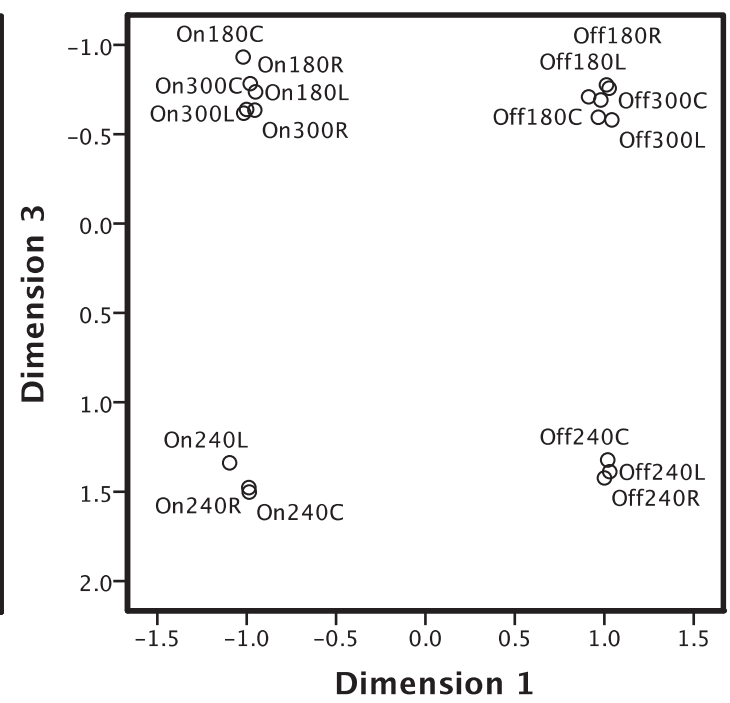

Fig. 4. The nonmetric weighted MDS (WMDS) 3-dimension solution for all participants' pairwise dissimilarity task (PDT) data, showing dimension 1 vs. dimension 2 in the left plot and dimension 1 vs. dimension 3 in the right plot. The points are labelled with a stimulus code: On/Off refers to the tremolo; 180/240/300 refers to the frequency; and L/C/R refers to the interaural presentation. 

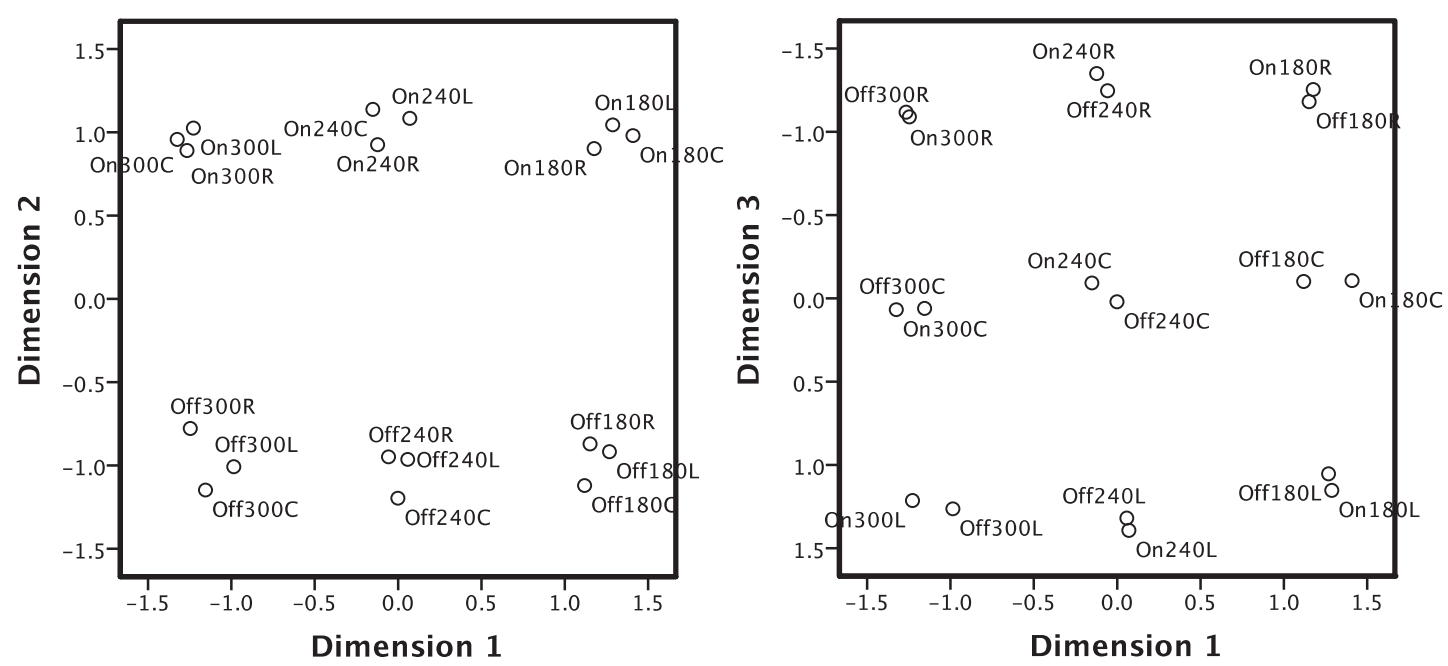

Fig. 5. The nonmetric weighted MDS (WMDS) 3-dimensional solution for all participants' projective mapping task (PMT) data, showing dimension 1 vs. dimension 2 in the left plot and dimension 1 vs. dimension 3 in the right plot. The points are labelled with a stimulus code: On/Off refers to the tremolo; 180/240/300 refers to the frequency; and L/C/R refers to the interaural presentation.

$300 \mathrm{~Hz}$ and $180 \mathrm{~Hz}$ grouped together at the top). The interaural presentation is not clearly differentiated in these results (or when the dimensions are rotated).

While metric and nonmetric WMDS of the PDT data allowed for the recovery of tremolo (on/off) and stimulus frequency $(180 \mathrm{~Hz}, 240 \mathrm{~Hz}, 300 \mathrm{~Hz})$, it did not successfully allow for the recovery of the interaural presentation. Therefore, it appears that WMDS analysis of the PDT data did not allow for the full recovery of the three dimensions.

\subsection{PMT Results}

The PMT data were analyzed separately for each participant using metric and nonmetric CMDS. An overview of the Stress values for solutions with 1 to 5 dimensions for each participant's results is shown in Fig. 1 (the faded lines in the two rightmost plots). The individual results show a clear knee point at two dimensions for the metric solution, indicating that a two-dimensional solution is optimal. This is expected: PMT limits the subject to a two-dimensional response format. The nonmetric results are less clear, although half the results still indicate a two-dimensional solution is optimal (although we know the participants identified three dimensions).

The PMT data from all participants were then analyzed using metric and nonmetric WMDS, and the stress values for solutions with 2 to 5 dimensions are shown by the dashed lines in the two rightmost plots of Fig. 1. Unlike the similar analyses for PDT, a knee point is apparent that indicates the three-dimensional solution to be optimal for both metric and nonmetric WMDS analyses of the PMT data.

The WMDS solution was also visually inspected, and it was apparent that three meaningful perceptual dimensions could be recovered from the PMT data. As can be seen in Fig. 5, the groupings/clusters of stimuli associated with dimension 1 correspond with stimulus frequency (high frequency to the left, low to the right). Dimension 3 corresponds with interaural presentation: Stimuli presented solely to the left ear correspond with low values on dimension 3, diotic stimuli are shown in the middle, and stimuli presented solely to the right ear are shown towards the top. The groupings/clusters associated with dimension 2 correspond with with tremolo. Stimuli with tremolo off are at the bottom, and those with tremelo on are at the top. As such, a WMDS analysis of the PMT data allowed for the full recovery of the three dimensions.

\subsection{Analysis-MFA}

The data resulting from PMT is usually analysed using MFA [26], a method developed to analyse data from a set of participants that can take into account a group structure defined by the experimenter [25]. As MFA is only appropriate for analyzing data from a set of participants, the separate analysis of the results for each participant discussed above was not undertaken.

The results from the MFA can be seen in Fig. 6 (upper plots for the PDT data and lower plots for the PMT data). It can be seen from the scree plot of explained variance for each dimension shown in Fig. 7 that, as for the MDS analysis, there is a clearer three-dimensional solution for the PMT data than the PDT data. The first two dimensions for the PDT data in Fig. 6 show clear differentiation of the tremolo on/off and stimulus frequency, respectively, similar to the MDS analysis shown in Fig. 4. In the MFA results (unlike the MDS), the interaural presentation can be seen to vary across the third dimension, though as a subset of the stimulus frequency: the main grouping is by frequency $(180 \mathrm{~Hz}$ and $300 \mathrm{~Hz}$ at the top and $240 \mathrm{~Hz}$ at the bottom), though within each of these groups there is a consistent order of interaural presentation ( $\mathrm{L}, \mathrm{C}, \mathrm{R}$, respectively, from top to bottom).

For the PMT data, three clear dimensions can be seen (dimension $1=$ stimulus frequency, dimension $2=$ tremolo on/off, and dimension $3=$ interaural presentation). This is 
a) Results for PDT
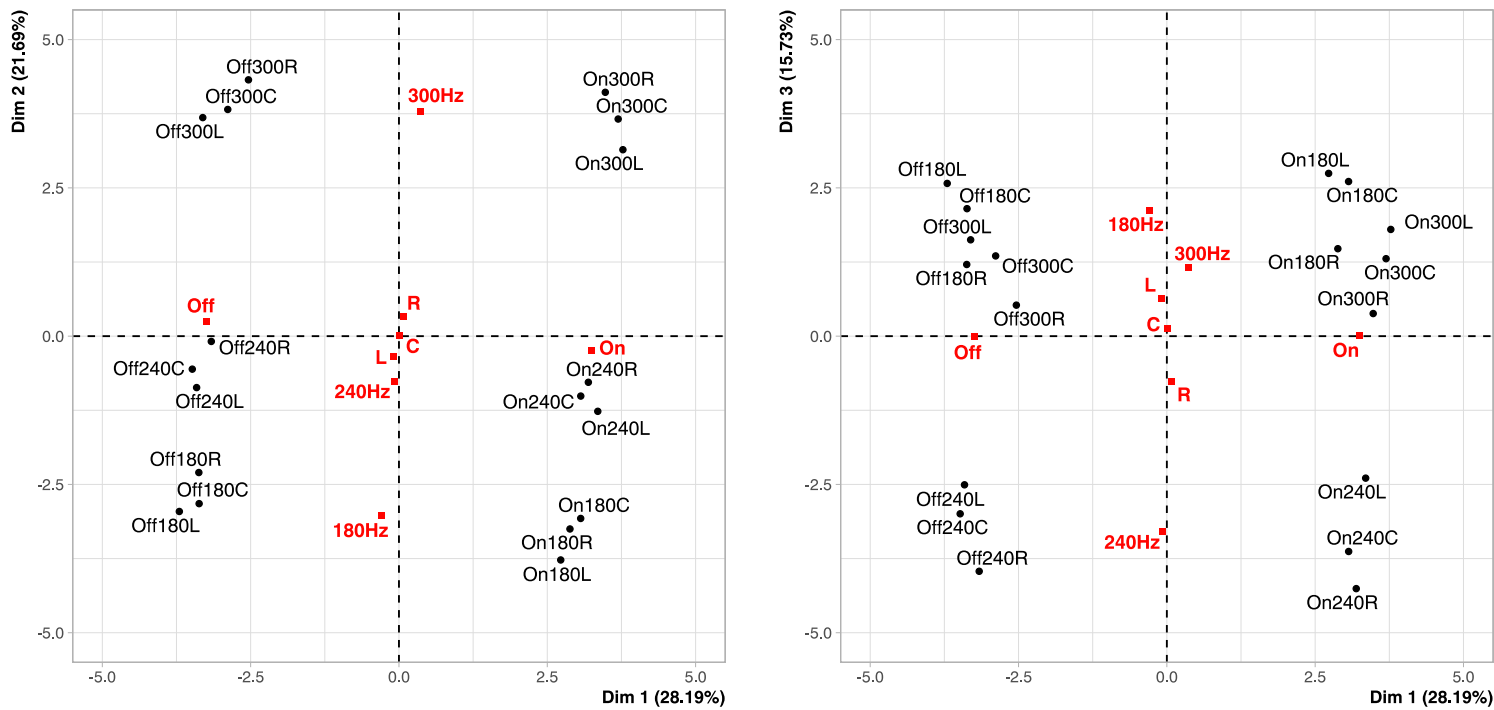

b) Results for PMT
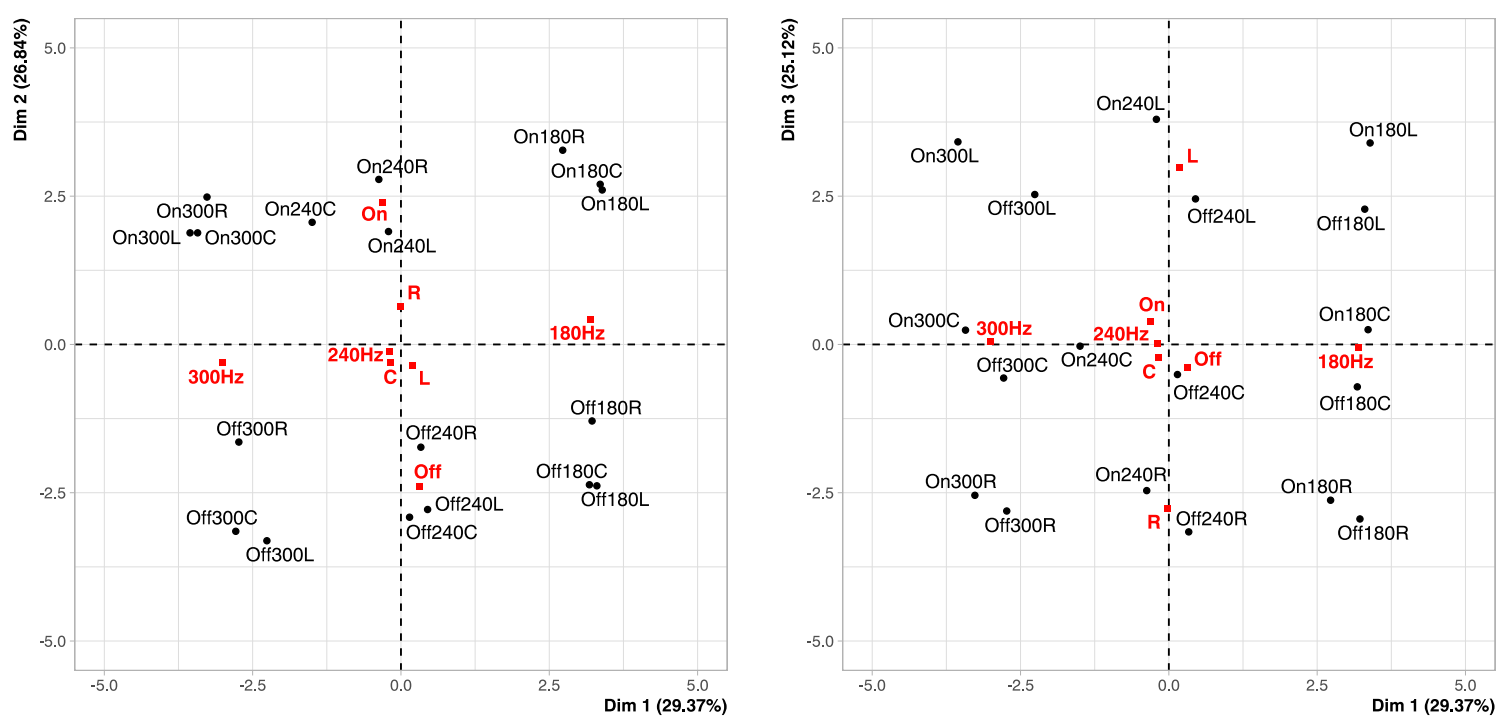

Fig. 6. The Multiple Factor Analyses (MFA) 3-dimension solution for all participants a) pairwise dissimilarity task (PDT) data (upper plots) and b) projective mapping task (PMT) data (lower plots), showing dimension 1 vs. dimension 2 in the left plots and dimension 1 vs. dimension 3 in the right plots. The points are labelled with a stimulus code: On/Off refers to the tremolo; 180/240/300 refers to the frequency; and L/C/R refers to the interaural presentation. The circular (black) points indicate results for individual stimuli, the square (red) points indicate the grouped results for each factor.

similar to the MDS analysis, though the dimension order is altered.

\subsection{Duration Results}

The duration results are shown as box plots in Fig. 8. It can be seen that the time taken for PMT was significantly less than that for PDT: the median values being 7 min $15 \mathrm{~s}$ for PMT and 31 minutes for PDT. In all cases, PMT took less time than PDT: the range of times taken for PMT was 5 $\mathrm{min} 10 \mathrm{~s}$ to $18 \mathrm{~min}$, whereas the range of time taken for PDT was $27 \mathrm{~min}$ to $35 \mathrm{~min}$. Hence, PMT took a median duration of approximately one-quarter of PDT, and even taking the smallest differences PMT still took only two-thirds the duration of PDT. PDT took over half an hour for most participants to complete; this duration is therefore towards the range for which participant fatigue may become an issue, though for this experiment is within the recommended limit of 30 to 40 min before a break is required [1, pp. 302].

\subsection{Discussion}

The dissimilarity results were analyzed primarily to determine whether three meaningful dimensions could be derived from analysis of the PDT and PMT data. This was undertaken using numerical and visual analysis methods. It was found that one participant's PDT data could be analyzed to recover three meaningful dimensions. However, it 

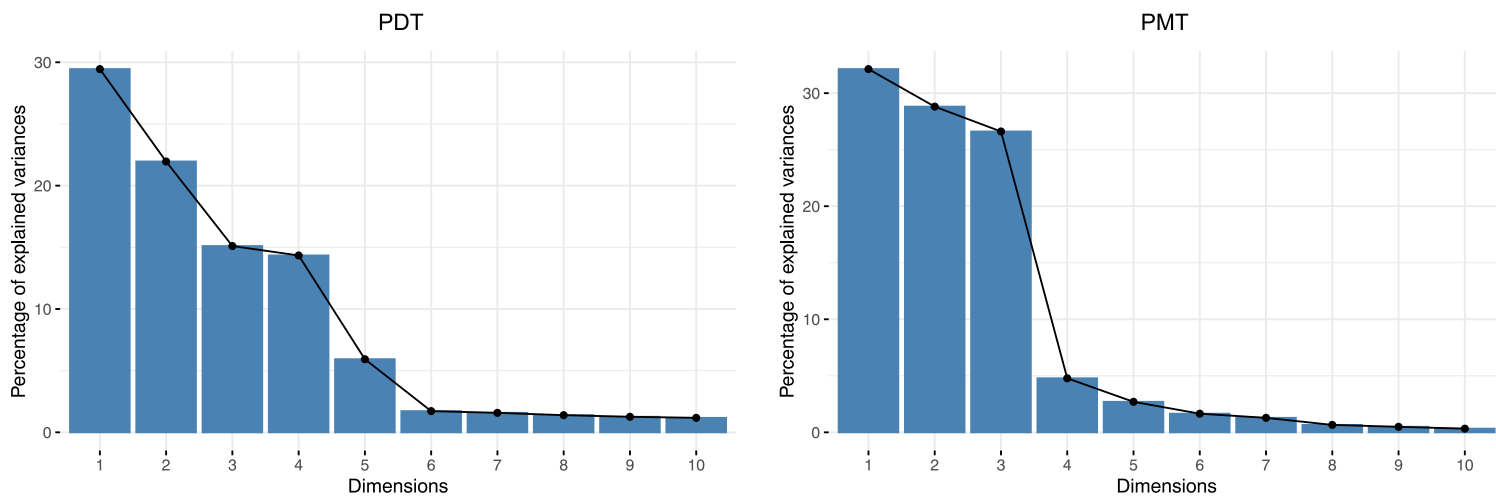

Fig. 7. The scree plot of explained variance for each dimension of the Multiple Factor Analyses (MFA), for the pairwise dissimilarity task (PDT) data (left plot) and the projective mapping task (PMT) data (right plot).

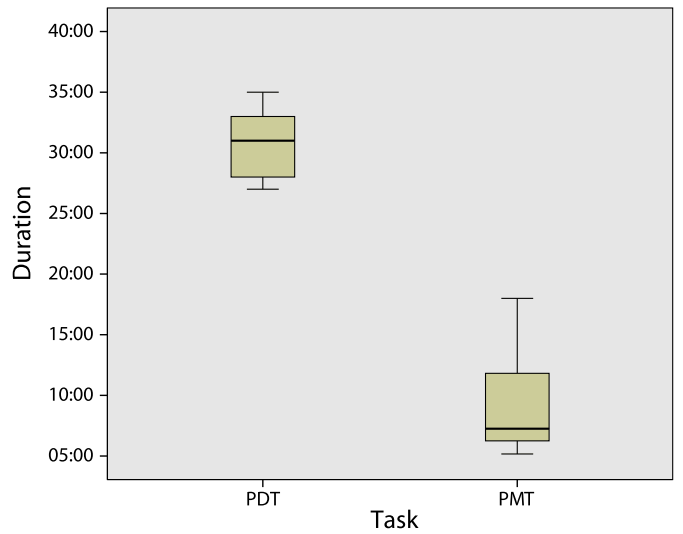

Fig. 8. Box plot of the task duration (min:s).

was not possible to determine three meaningful dimensions from the WMDS analysis of the aggregated PDT data from all participants, and only a weak third meaningful dimension could be determined using MFA. This is surprising given that (a) as discussed above, the ability of PDT to enable derivation of more than two meaningful dimensions is not generally considered to be limited and (b) the stimuli were intentionally unambiguous, and all participants were explicitly informed about the nature of the stimuli.

Discussions with the participants after the test indicated that all three stimulus dimensions were clear and identifiable for all participants, as was intended. So why could the analysis not recover three dimensions from the PDT data?First, it may be that the subjects failed to adopt an effective strategy for PDT; future research ought to investigate whether repeating the task or training the participants can meaningfully affect this. Second, it may be that there was too much variation between the strategies used by each participants, leading to insufficient commonality when recovering the full dimensionality. Finally, it may be that the perceptual magnitude of the stimulus frequency and tremolo dimensions was much larger than that of the interaural presentation. This could result in the differences of the former being emphasized in the responses and the interaural presentation judgments becoming of similar magnitude to the judgment error or noise. This is considered unlikely given that all three dimensions could be retrieved from the PMT data; however, it would still be of interest to repeat this experiment with other stimulus sets.

For the PMT data, as expected, only two dimensions could be derived from each participant's responses with CMDS. However, WMDS and MFA were able to successfully derive three meaningful dimensions from the PMT data from the group of participants. Hence, it appears that PMT does not restrict the resulting solution to two dimensions, as long as multiple responses are aggregated in the analysis.

It is of interest to further consider why more than two dimensions could be recovered from a 2-dimensional response format. Examination of each individual participant's results indicates that the participants attempted to communicate the three perceptual dimensions using a range of varied strategies, as has been found previously [27]. Most participants tended to use subcategories to express the third dimension, such as the examples shown in Fig. 9(a) and Fig. 9(b). In Fig. 9(a), dimension 1 is tremolo on/off, and dimension 2 is stimulus frequency. The interaural presentation is expressed as a subcategory of dimension 1. In Fig. 9(b), dimension 1 is the interaural presentation, dimension 2 is tremolo on/off, and stimulus frequency is a subcategory of dimension 2.

While the subcategory representation was most common, there were other representations used. For example, participant 6 (shown in Fig. 9(c)) employed a radial representation in which dimension 1 related to the stimulus frequency, dimension 2 related to the interaural presentation, and tremolo on/off was represented by the distance from the center; a similar strategy was found previously [11]. A final strategy worth noting was one employed by participant 8 (shown in Fig. 9(d)). This appears to combine a radial and a subcategory layout, with stimulus frequency represented on dimension 1 , interaural presentation represented on dimension 2 , and tremolo on/off represented as a radial subcategory.

It appears, therefore, that a range of strategies were employed for PMT, and there were different layouts within these strategies. For the subcategory representations, the attribute chosen to be represented as a subcategory differed 
a)

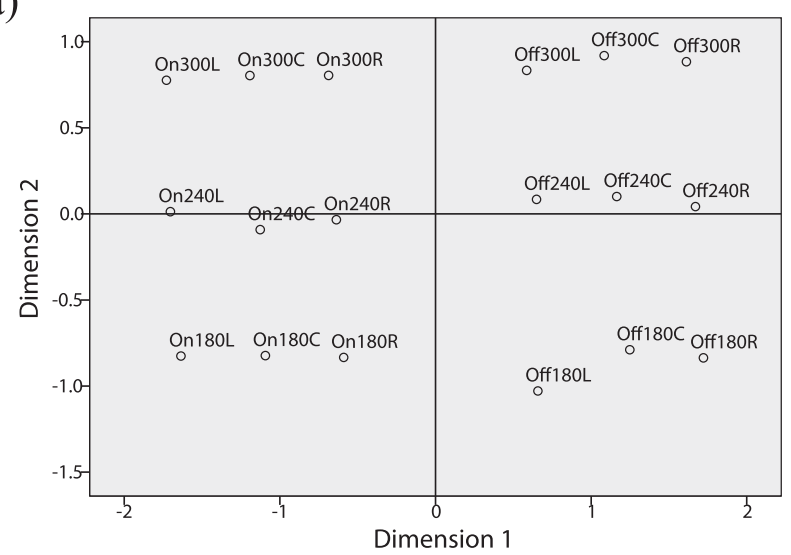

c)

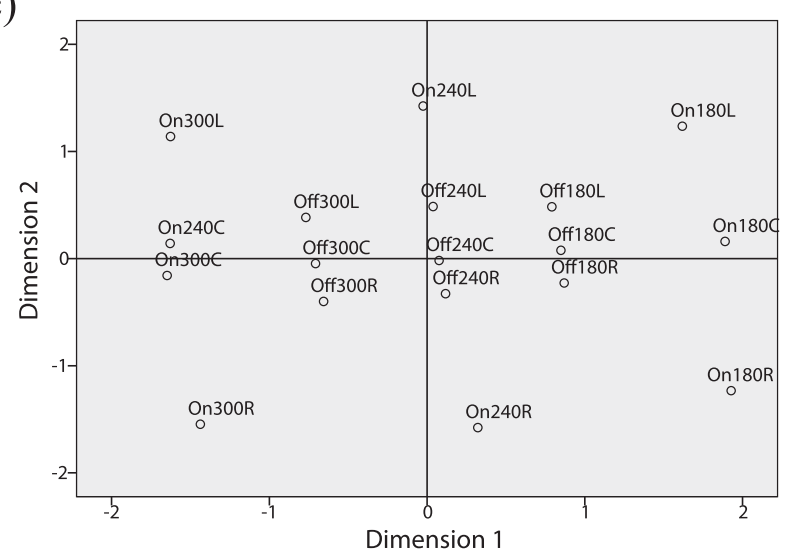

b)

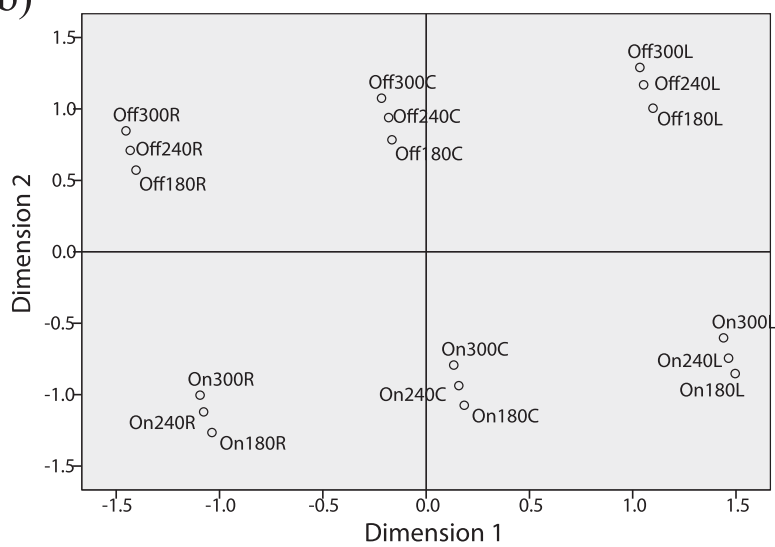

d)

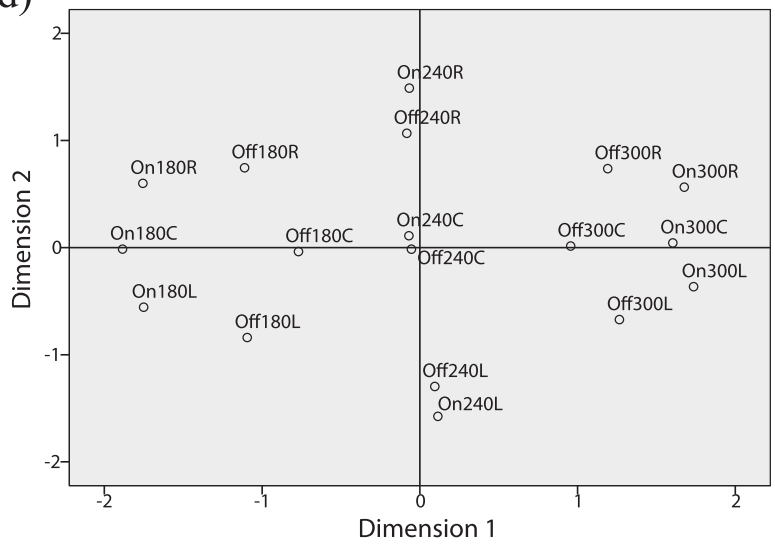

Fig. 9. Example 2-dimensional Multi-Dimensional Scaling (MDS) analyses from the projective mapping task (PMT) data of 4 participants: a) participant 5; b) participant 10; c) participant 6; and d) participant 8.

(e.g., interaural presentation for Fig. 9(a) and stimulus frequency for Fig. 9(b)). Also, differing methods were selected for representing the subcategories (e.g., vertical, horizontal, or radial). Finally, not all participants used a subcategory representation. Hence, three dimensions could be retrieved using WMDS analysis of the PMT because of the differences between the strategy used in each participant's responses.

\subsection{Limitations and Further Work}

The sample size limits the confidence in the interpretation of the results; it may be that with additional participants a meaningful 3-dimensional solution could be found using PDT. However, given that all participants fully identified the dimensionality of the stimuli (indeed, they were told what the dimensionality was at the start of the test), and given also that the WMDS and MFA successfully recovered the dimensionality of the data for the PMT, in some ways, the small sample size speaks for the efficacy of recovery. In other words, it might be argued that PMT allowed for full recovery of the dimensionality in spite of the limited sample size.

A further limitation relates to the generalizability of our results, given the nature of the experimental design. The fact that listeners were informed a priori of the number and nature of the perceptual dimensions helped us to isolate potential sources of limitation in the methodology. However, in most real-world applications, the purpose of PDT and PMT is to provide information about the perceptual dimensions without priming the participants beforehand. Additionally, the nature of the stimuli for these experiments were purposefully chosen to be perceptually unambiguous. It therefore remains to be seen as to how well PMT translates to real-world problems in which the stimuli vary according to more complex perceptual dimensions.

\section{CONCLUSIONS}

This paper compared two methods of generating data for analysis: PDT and PMT. The aims were to determine the following: whether more than two dimensions can be recovered for data derived using PMT; how participants respond when limited to PMT's inherently two-dimensional response format; the relative time required to complete the two tasks; and, hence, whether PMT is an appropriate method for collecting data in experiments using auditory stimuli.

It was found that three meaningful dimensions could be recovered using WMDS and MFA for data generated using PMT. However, it was not possible to recover three 
clear meaningful dimensions for data derived using PDT, apart from CMDS analysis of one particular participant's responses. This result was unexpected because (a) limits on dimensionality are most commonly discussed for PMT rather than PDT, and (b) the participants were responding to intentionally unambiguous and orthogonally varying stimuli.

The PMT responses were examined, and it was found that in most cases, participants developed a strategy to express the three dimensions despite the two-dimensional response format. In some cases, this was through the use of subcategories; in other cases, radius was used as a dimension. The fact that the strategies differed allowed the three dimensions to be derived when all participants' responses were combined. It was found that PDT took the subjects much longer than PMT, with a median ratio of $4: 1$.

Hence, as PMT is more rapid, and does not appear to restrict the derivation of three dimensions any more than PDT (and in this experiment was more successful for the derivation of three meaningful dimensions than PDT), it is apparent that PMT is a viable method for collecting data for MDS and MFA in experiments using auditory stimuli.

\section{REFERENCES}

[1] S. Bech and N. Zacharov, Perceptual Audio Evaluation: Theory, Method and Application (John Wiley and Sons, Chichester, England, 2006).

[2] H. Abdi and D. Valentin, "Some New and Easy Ways to Describe, Compare, and Evaluate Products and Assessors," in D. Valentin, D. Z. Nguyen, and L. Pelletier (Eds.), New Trends in Sensory Evaluation of Food and Non-Food Products., pp. 5-18 (Vietnam National University, Ho Chi Minh, Vietnam, 2007).

[3] L. Vidal, R. S. Cadena, S. Correa, R. A. Abalos, G. Gomez, P. Varela A. Gimenez, and G. Ares, "Assessment of Global and Individual Reproducibility of Projective Mapping With Consumers," Journal of Sensory Studies, vol. 29, no. 1, pp. 74-87 (2014 Feb.), http://dx.doi.org/10.1111/joss.12083.

[4] J. Pagès, "Collection and Analysis of Perceived Product Inter-Distances Using Multiple Factor Analysis: Application to the Study of 10 White Wines From the Loire Valley," Food Quality and Preference, vol. 16, no. 7, pp. 642-549 (2005 Oct.), https://doi.org/ 10.1016/j.foodqual.2005.01.006.

[5] S. S. Schiffman and T. W. Knecht, "Basic Concepts and Programs for Multidimensional Scaling," in C. T. Ho and C. H. Manley (Eds.), Flavor Measurement, pp. 133157 (Marcel Dekker Inc., New York, New York, 1993).

[6] M. L. Davison, C. S. Ding, and S. Kim, "Multidimensional Scaling," in G. R. Hancock and R. O. Mueller (Eds.), The Reviewer's Guide to Quantitative Methods in the Social Sciences, pp. 265-280 (Routledge, New York, New York, 2010).

[7] C. Tang and H. Heymann, "Multidimensional Sorting, Similarity Scaling and Free Choice Profiling of Grape Jellies," Journal of Sensory Studies, vol. 17, no.
6, pp. 493-509 (2007 May), http://dx.doi.org/10.1111/ j.1745-459X.2002.tb00361.x.

[8] R. Popper and H. Heymann, "Analyzing Differences Among Products and Panelists by Multidimensional Scaling," in T. Næs and E. Risvik (Eds.), Multivariate Analysis of Data in Sensory Science, vol. 16 (Elsevier, Amsterdam, the Netherlands, 1996), http://dx.doi.org/10.1016/ S0922-3487(96)80030-X.

[9] V. R. Rao and R. Katz, "Alternative Multidimensional Scaling Methods for Large Stimulus Sets," Journal of Marketing Research, vol. 8, no. 4, pp. 642-649 (1971), http://dx.doi.org/10.2307/3150241.

[10] C. Delholm, P. B. Brockhoff, L. Meinert, M. D. Aaslyng, and W. L. Bredie, "Rapid Descriptive Sensory Methods-Comparison of Free Multiple Sorting, Partial Napping, Napping, Flash Profiling and Conventional Profiling," Food Quality and Preference, vol. 26, no. 2, pp. 267-277 (2012 Dec.), https://doi.org/10.1016/ j.foodqual.2012.02.012.

[11] M. Nestrud and H. Lawless, "Recovery of Subsampled Dimensions and Configurations Derived From Napping Data by MFA and MDS," Attention, Perception, and Psyhcophysics, vol. 73, pp. 1266-1278 (2011), http://dx.doi.org/10.3758/s13414-011-0091-0.

[12] M. C. King, M. A. Cliff, and H. W. Hall, "Comparison of Projective Mapping and Sorting Data Collection and Multivariate Methodologies for Identification of Similarity-of-Use of Snack Bars," Journal of Sensory Studies, vol. 13, pp. 347-358 (2007 May), http://dx.doi.org/10.1111/j.1745-459X.1998.tb00094.x.

[13] G. P. Scavone, S. Lakatos, and C. R. Harbke, "The Sonic Mapper: An Interactive Program for Obtaining Similarity Ratings With Auditory Stimuli," Proc. International Conference on Auditory Display (2002 Jul.).

[14] E. Risvik, J. A. McEwan, and M. Rødbotten, "Evaluation of Sensory Profiling and Projective Mapping Data," Food Quality and Preference, vol. 8, no. 1, pp. 63-71 (1997 Jan.).

[15] S. S. Schiffman, M. L. Reynolds, and F. W. Young, Introduction to Multidimensional Scaling: Theory, Methods, and Applications (Academic Press, New York, New York, 1981).

[16] D. Giacalone, M. Nitkiewicz, S. Moulin, T. Booason, J. Laugesen, and S. Bech, "Sensory Profiling of HighEnd Loudspeakers Using Rapid Methods-Part 2: Projective Mapping With Expert and Naive Assessors," presented at the 142nd Convention of the Audio Engineering Society (2017 May), convention paper 9775.

[17] T. Letowski, "Sound Quality Assessment: Concepts and Criteria," presented at the 87th Convention of the Audio Engineering Society (1989 Oct.), convention paper 2825, https://doi.org/10.1121/1.417683.

[18] R. Melara and L. Marks, "Interaction Among Auditory Dimensions: Timbre, Pitch, and Loudness," Perception and Psychophysics, vol. 48, no. 2, pp. 169-178 (1990 Sep.).

[19] F. Rumsey, S. Zielinski, R. Kassier, and S. Bech, "Relationships Between Experienced Listener Ratings of Multichannel Audio Quality and Naive Listener 
Preferences," Journal of Acoustical Society of America, vol. 117, no. 6, pp. 3832-3840 (2005 Jul.), http://dx.doi.org/10.1121/1.1904305.

[20] T. Neher, T. Brookes, and F. Rumsey, "A Hybrid Technique for Validating Unidimensionality of Perceived Variation in a Spatial Auditory Stimulus Set," $J$. Audio Eng. Soc., vol. 54, no. 4, pp. 259-275 (2006 Apr.), http://epubs.surrey.ac.uk/id/eprint/815232.

[21] K. Kurozumi and K. Ohgushi, "The Relationship Between the Cross-Correlation Function of Two-Channel Acoustic Signals Reproduced Through the Headphones and Spatial Impressions of Sound Image," Journal of the Acoustical Society of America, vol. 78, no. S1, p. S19 (1985), http://dx.doi.org/10.1121/1.2022680.

[22] O. Macherey and A. Delpierre, "Perception of Musical Timbre by Cochlear Implant Listeners: A Multidimensional Scaling Study," Ear and Hearing, vol. 34, no. 4, pp. 426-436 (2013 Jul./Aug.), http://dx.doi.org/10.1097/AUD.0b013e31827535f8.
[23] T. M. Elliott, L. S. Hamilton, and F. E. Theunissen, "Acoustic Structure of the Five Perceptual Dimensions of Timbre in Orchestral Instrument Tones," Journal of the Acoustical Society of America, vol. 133, no. 1, pp. 389-404 (2013 Jan.), http://dx.doi.org/10.1121/1.4770244.

[24] B. F. Blake, S. Schulze, and J. M. Hughes, "Perceptual Mapping by Multidimensional Scaling: A Step by Step Primer," Research Reports in Consumer Behavior (2003).

[25] J. Pagès, Multiple Factor Analysis by Example Using $R$ (CRC Press, Boca Raton, Florida, 2014).

[26] S. Lê and T. Worch, Analyzing Sensory Data With $R$ (CRC Press, Boca Raton, Flordida, 2014).

[27] C. Savidan and C. Morris, "Panelists' Performances and Strategies in Paper-Based and ComputerBased Projective Mapping," J. of Sensory Studies, vol. 30, no. 2, pp. 145-155 (2015), http://dx.doi.org/10.1111/joss. 12146.

\section{THE AUTHORS}

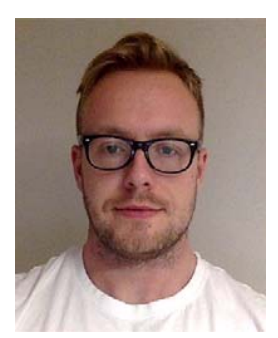

Matthew Vowels

Matthew Vowels originally graduated from the Tonmeister course at the University of Surrey, United Kingdom, in 2012. Since then, he has been working as an electroacoustic design engineer and project manager for an audio technology company and has also been working as an associate tutor in electroacoustics at the University of Surrey. He has an MSc in Computer Vision, Robotics and Machine Learning and is currently a $\mathrm{PhD}$ student in machine learning at the Centre for Computer Vision, Speech, and Signal Processing at the University of Surrey.

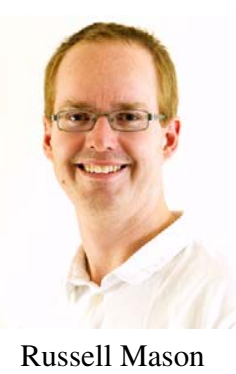

Russell Mason was awarded a $\mathrm{PhD}$ in audio engineering and psychoacoustics from the University of Surrey in 2002 and is currently a senior lecturer in the Institute of Sound Recording, University of Surrey, with over 100 published journal and conference papers. His research interests are focused on psychoacoustic engineering, and he has led the development of subjective evaluation methods and computational models of aspects of auditory perception, for application in spatial audio, evaluation of timbre, source separation, and personal sound zones. 